\title{
New Worlds Observer Telescope and Instrument Optical Design Concepts
}

\author{
Joseph M. Howard", Charlie Noecker", Steve Kendrick`, \\ Steve Kilston", Bruce Woodgate", Webster Cash" \\ " NASA Goddard Space Flight Center, Greenbelt, MD, USA \\ - Ball Aerospace Technology Corp, Boulder, CO, USA \\ `University of Colorado, Boulder, CO, USA
}

\begin{abstract}
Optical design concepts for the telescope and instrumentation for NASA's New Worlds Observer program are presented. A four-meter multiple channel telescope is discussed, as well as a suite of science instrument concepts. Wide field instrumentation (imager and spectrograph) would be accommodated by a three-mirror-anastigmat telescope design. Planet finding and characterization, and a UV instrument would use a separate channel that is picked off after the first two mirrors (primary and secondary). Guiding concepts are also discussed.
\end{abstract}

Keywords: Optical design, Optical Modeling, Terrestrial Planet Finding, Three-Mirror-Anastigmat

\section{INTRODUCTION}

The New Worlds Observer (NWO) is a mission to find and study the planets that circle our neighboring stars. It will find hundreds of nearby planets, some Earth-like, and classify each through spectroscopy. NWO will then move beyond exploration to perform detailed studies of planetary formation, evolution, surfaces, and atmospheres. It will search for habitable worlds, identify water planets like our home world, and look for the biomarkers that may indicate life. The New Worlds Observer will support a broad range of fundamental new science and exploration. The leap in capability is such that, no matter how much exo-planet science advances in the coming decade, its impact will be undiminished.

NWO is a large class exo-planet mission that employs two spacecraft: a "starshade" to suppress starlight before it enters the telescope and a conventional telescope to detect and characterize exo-planets (See Figure 1). It represents a new and exciting architecture for exo-planet detection that can be matured rapidly and is the only currently affordable approach to finding significant numbers of terrestrial planets in the habitable zones of Sun-like stars. It has much higher efficiency and much broader spectral coverage, making it the only viable approach to classifying and studying such planets once found.

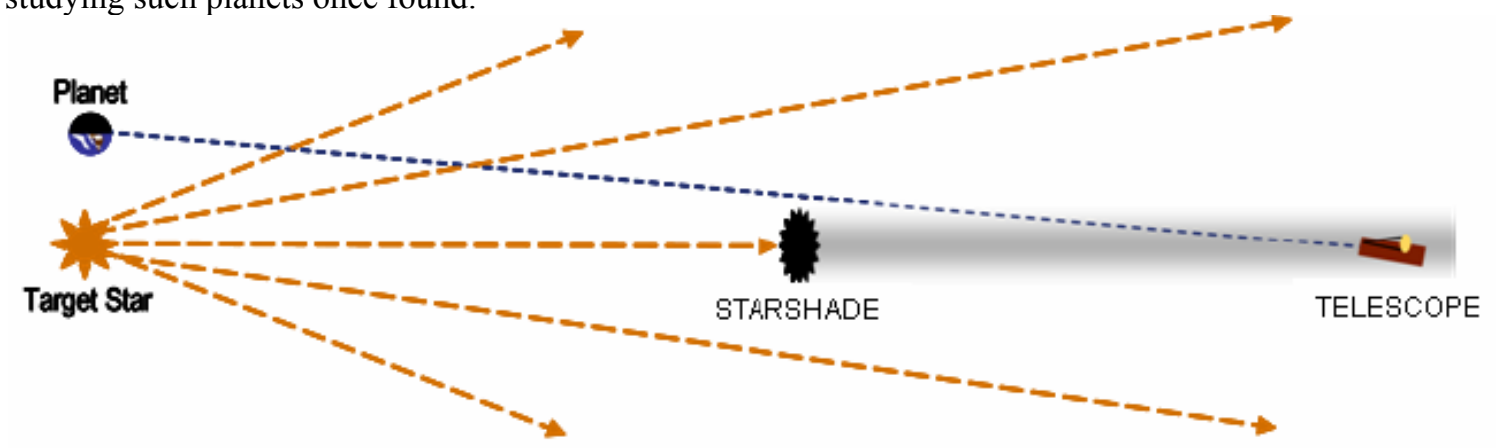

Figure 1. NWO throws a deep shadow over the telescope, reducing starlight intensity by more than 1010, but allows planet light to be viewed. 
Instrument concepts are summarized in Section 2. Their influence on the choice of telescope design is discussed in Section 3, followed by the introduction and design of a "dual channel" telescope in Section 4. Concluding remarks are offered in Section 5.

\section{INSTRUMENT CONCEPTS}

The instrument complement for NWO naturally follows closely the instruments that were recommended for the Terrestrial Planet Finder - Coronagraph Mission (TPF-C). See for example the TPF-C Instrument Concept Study reports for CorECam [1], CorSpec [2], and Mag30Cam [3]. The NWO Instruments currently being considered are the Exoplanet Camera (ExoCam), the ExoPlanet Spectragraph (ExoSpec), the General Astrophysics Wide-Field Camera (GA/WFcam), and the Ultra-Violet Instrument (UV Inst).

\section{ExoCam}

Initial detection of planets calls for a sensitive broadband detector system, comprising color filters and a modestsized CCD. We will use the widest possible optical bandwidth to allow quick extraction of faint point-like sources among background signals from local- and exo-zodiacal dust and the speckles of stellar leakage. In the present understanding, internal-occulter coronagraphs achieve sufficient stellar-leakage suppression only within an optical bandwidth of order 20-25\%; a simple color filter in front of the CCD suffices to exclude wavelengths outside this range. Corresponding bandwidth limitations for an external starshade like NWO's appear to be much looser: currently we find there is adequate suppression over the full $0.4-1.0 \mu \mathrm{m}$ passband of a typical CCD's quantum efficiency. In this case, no filter would be needed. However, a filter wheel is useful for other reasons, as described below, and it is easy to provide.

A four-meter aperture is currently baselined for the telescope in order to meet both the sensitivity and astrometry needs of the mission. The camera CCD should critically sample the point spread function near $\lambda=500 \mathrm{~nm}(\lambda / 2 \mathrm{D}=$ 13 mas for $\mathrm{D}=4 \mathrm{~m}$ ). This gives a near-optimum detection sensitivity for wavelengths with the best detection IWA. This sampling criterion can be adjusted to mitigate a loss of sensitivity at longer wavelengths. The CCD should also span the full detectable planetary system, including all its outer planets. The external occulter system has an essentially unlimited outer working angle, so the CCD could be extended to span 3-5 arcseconds (up to $400 \times 400$ pixels). In practice the outer planets will become too faint to detect before then, and their orbits will evolve too slowly to be measured well in a 5 year mission. Limitations like these will govern the CCD's useful size.

\section{ExoSpec}

After detection of a planet candidate, we would immediately want to confirm it as a planet and exclude faint background stars or galaxies, which are expected to be common. The first phase of confirmation would be a threeband color determination, i.e. a low-resolution spectrum of the object. This requires color filters with a bandwidth of about $26 \%$, together spanning the range from $\sim 0.5-1.0 \mu \mathrm{m}$.

After the object is confirmed with reasonably high probability to be a planet, the detailed characterization calls for collecting a full spectrum at a spectral resolution $\mathrm{R} \approx 70$. This enables detection of key molecular species in the planet's atmosphere: $\mathrm{O}_{2}(0.76 \mu \mathrm{m})$ and $\mathrm{H}_{2} \mathrm{O}(0.94 \mu \mathrm{m})$. The baseline spectrometer should be capable of detecting these lines if the IWA allows it. Also, a helpful enhancement would be the ability to detect $\mathrm{CO}_{2}(1.06 \mu \mathrm{m}$ and longer).

Ideally the spectrometer should cover the planet PSF with critically sampling spatial resolution, similar to the broadband detector. An integral field spectrometer (IFS) is a useful instrument type for this purpose, generating a spectrum for each pixel in a grid spanning an area on the sky. For the external occulter, the telescope boresight can be repointed, independent of the stellar suppression; so it is possible to aim the IFS pixels at a known planet, and thus the IFS could be made barely large enough to span the planet PSF. But anticipating that each star will harbor multiple planets that we'll want to characterize. Therefore it would be better to cover the entire planetary system with the IFS if possible, or at least a substantial fraction of it. Like the broadband detector, we want the IFS to be critically sampling at $\lambda=500 \mathrm{~nm}$, with a span up to $3-5$ arcsec. 
By analogy with earlier coronagraphs, we think a CCD is adequate for this instrument as well. But at a spectral resolution of $\mathrm{R}=70$, the per-pixel flux is very low; to maintain high $\mathrm{SNR}$, it would help to use a detector with low read noise, such as a photon-counting array detector.

\section{GA/WFCam}

The telescope can accommodate a very wide field camera if we want it. It is plausible to contemplate a $10 \times 20$ arcminute field which is nearly diffraction limited and critically sampled at $\lambda=500 \mathrm{~nm}$ (pixel width $=\lambda / 2 \mathrm{D}=13 \mathrm{mas}$ ). This calls for about $4 \times 10^{9}$ pixels, much larger than the largest focal planes built to date, but scalable from instruments such as Kepler [4]. If the cost is too high for such an expanse of pixels, or the downlinking its data proves too much of a burden, a $3 \times 3$ arcminute (or rectangular, e.g. $1 \times 10$ ) focal plane system would still enable a rich variety of general astrophysics studies, including transiting and astrometric planet searches around background stars, supernova detections, Galactic stellar population studies, galaxy evolution, dark matter mapping, tracing the cosmic history and equation of state of dark energy, and cosmology.

Another natural addition to consider is a near-IR focal plane of comparable extent. At the longer wavelengths, the pixel size would be larger and the pixel count smaller by at least a factor 2. Ideally the IR is separated from the visible light by dichroics, so the visible and IR fields can overlap substantially or entirely.

Some type of wide field camera seems necessary in any case, to capture a sufficient number of background stars for the telescope pointing control. We want high-quality pointing control in almost any patch of sky, both for faintobject general astrophysics (as outlined above) and for exoplanet observations when the host star has been blocked by the starshade. A larger wide-field camera allows brighter guide stars and better control performance; at 10 arcminute $^{2}$ field, this limit would be about $\mathrm{V}=16-18$.

\section{UV Inst}

A faint object spectrograph in the UV is being considered to compliment the science capability of NWO. It would be a design derived from the Cosmic Origins Spectrograph on Hubble (HST/COS), and would require only a small field of about 3 arc seconds in diameter. The design should provide a spectral resolution of $\mathrm{R} \approx 20,000$ over a wide spectral range of $115-350 \mathrm{~nm}$. Finally, the detector for such an instrument would likely be a microchannel plate, of approximate size $16,000 \times 1000$ pixels.

\section{TELESCOPE CONCEPTS}

As discussed in the previous section, three of the four instruments (ExoCam, ExoSpec, UV Inst) have very modest field of view requirements, at most being 20 arc-seconds square. The GA/WFcam, however, requires more than an order of magnitude increase in field, as illustrated in Figure 2.
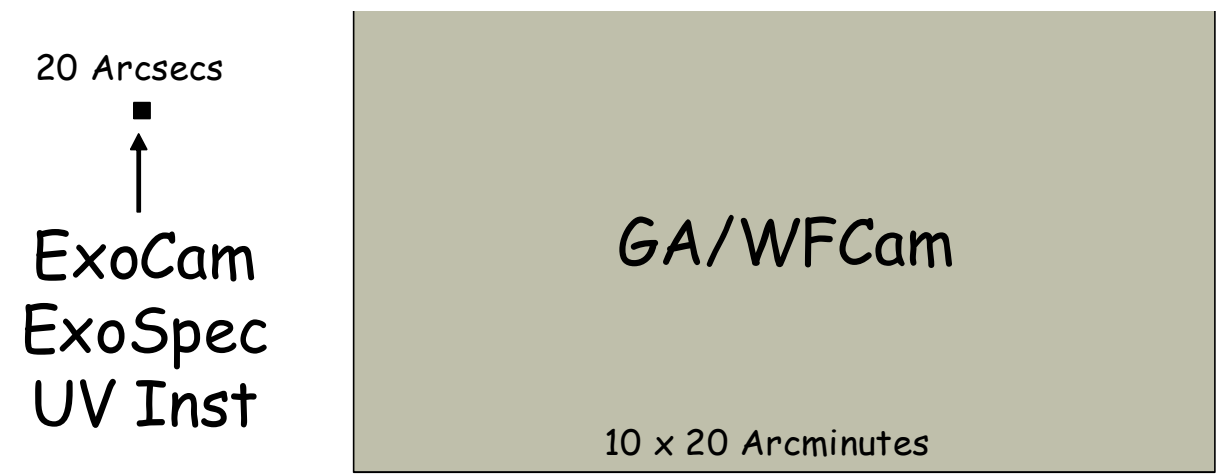

Figure 2. Relative Field of View sizes for NWO Instrumentation 
Since NWO requires a large corrected field of view with a large bandpass, the natural design choice for the mission is a three-mirror design, such as Korsch's three-mirror-anastigmat (TMA) [5]. This design employs a conic surface on three mirrors to correct third-order spherical, coma, and astigmatism aberrations, and typically a fold mirror to return the light behind the primary mirror. Petzval curvature can also be corrected, or made flat, by proper choice of the powered mirror curvatures. When such constraints are in place, the telescope can be optimized to deliver highly corrected imagery over a wide field of view onto a flat detector. Two-mirror telescopes, on the other hand, can only correct two of the third-order aberrations, typically spherical and coma. This limits the field of usable field of view of such designs to typically under a square arc minute without further correction. Figure 3 illustrates the two different types of telescopes which are nominally sufficient for each of the instruments.

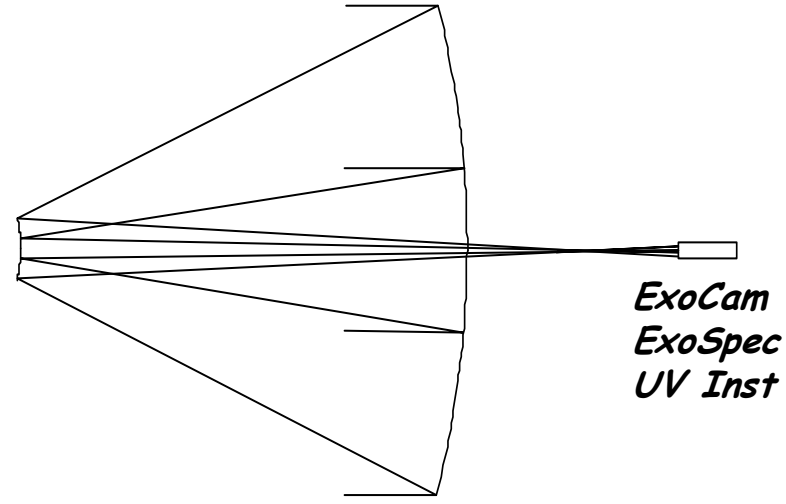

Two-Mirror Cassegrain

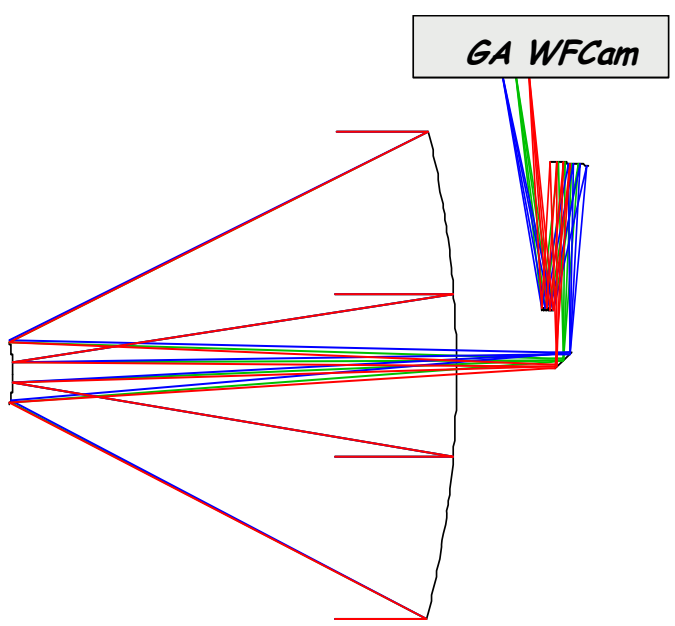

Three-Mirror-Anastigmat (TMA)

Figure 3. Nominal telescope design form for the NWO instrumentation

As stated, choosing a TMA design for the NWO telescope ensures that the GA/WFCam can meet the necessary image quality performance over its field of view. The smaller field instruments, however, will result in a degraded throughput, or sensitivity, due to the additional (and unnecessary) surfaces used to correct and fold the system. Fortunately, it is possible to take advantage of the field bias typically employed in the TMA design form. As seen in Figure 3, the wide field of the TMA necessitates a bias in the field of regard so that no self-obstruction occurs between the third and fourth reflections. A separate channel for the TMA telescope on the axis of the primary and secondary mirrors can be used. This "dual channel" concept is illustrated in Figure 4, where the small field instruments are held on the axis of the telescope, while the GA/WFCam defines the TMA itself. The primary (PM), secondary (SM), and tertiary (TM) mirrors are all labeled in the figure, as well as the internal focus located between the SM and TM, and a fold mirror (FM) located at the exit pupil of the telescope. The internal focus will also be referred to as the Cassegrain Focus (CF), since it follows the two-mirror front end of the TMA. This "dual channel" configuration allows for several advantages, including the increased sensitivity due to fewer losses at a reduced number of reflections, cleaner optical interfaces between the telescope and the instruments, and even employing customized coatings for shorter wavelengths only in the primary and secondary mirrors for the UV Instrument. 


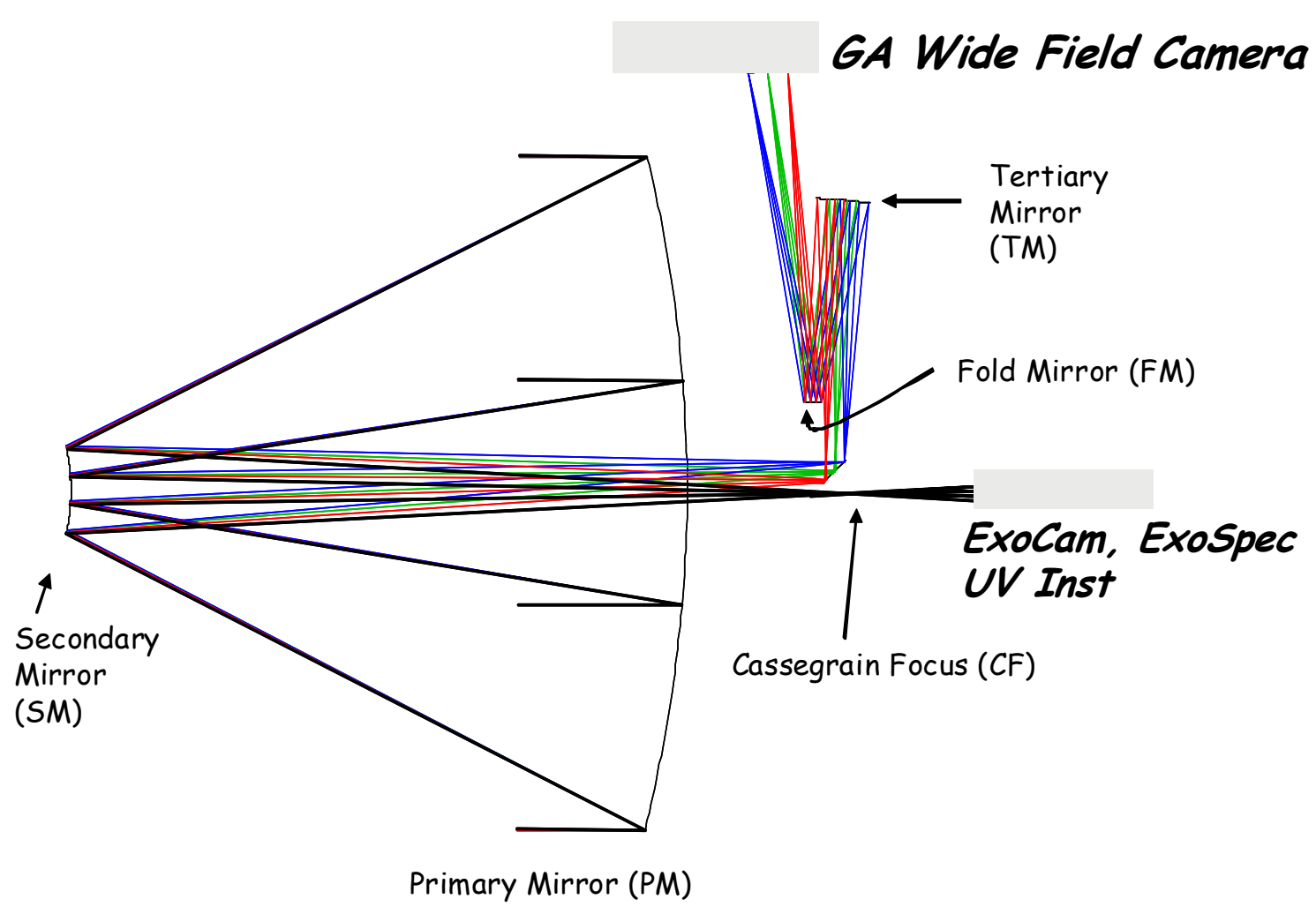

Figure 4. "Dual Channel" Telescope for the New Worlds Observer

\section{OPTICAL DESIGN OF THE “DUAL CHANNEL” TELESCOPE}

The challenge for designing the dual channel telescope is to have both good image quality at the TMA focal surface as well as the Cassegrain focal surface (CF). Furthermore, the natural layouts of the channels are such that the CF pickoff will be on-axis (i.e. on the axis defined by the PM and SM), since the aberrations there are the least, and that the large field will be off-axis, since self-obstruction occurs and a fold mirror will be employed to keep light behind the primary mirror. Designing a "dual channel" telescope is straightforward. A four-step process is sufficient:

1) Design a TMA for the large field of view required by the observatory.

2) Vary the PM radius and conic to optimize image quality at the fixed CF.

3) Vary the TM radius and conic to improve the degraded image quality at the TMA.

4) Add an aspheric corrector surface the fold-mirror (FM) located at the TMA exit pupil.

In step 1, one can either search the literature for existing designs and modify them, or just start from scratch. Automated methods exist if you choose the latter, such as employing SLIDERS [6] which can be used on the freeware version of OSLO [7]. For the purposes of this paper, our "starting design" will be the f/20 4-meter aperture TMA illustrated in Figure 4, which was designed using SLIDERS. As with most astronomical telescopes, the aperture stop is located at the primary mirror.

The performance of the starting system at the TMA focal surface is illustrated in Figure 5. On the left of the figure is a two-dimension plot showing the root-mean-square wave-front error (RMS WFE) at the center, corners, and sides of the 10x20 arc-minute field of view (FOV). The axes on the plot are the field in arc-minutes, and the values on the plot are nm RMS WFE. On the right side of the figure are the wave-front error maps (WFE), or pupil maps, for each of the nine fields. Note that the aspect ratio of the FOV is $2 \times 1$, even though the WFE maps appear in a square in the right portion of Figure 5. Each of the WFE maps is plotted on the same scale, so one can deduce the 
type of error (i.e. coma, spherical, etc.) as well as the relative magnitude. For the nominal system, it is clear from Figure 5 that the system is dominated by coma and astigmatism at the outermost (i.e. upper) field corners.
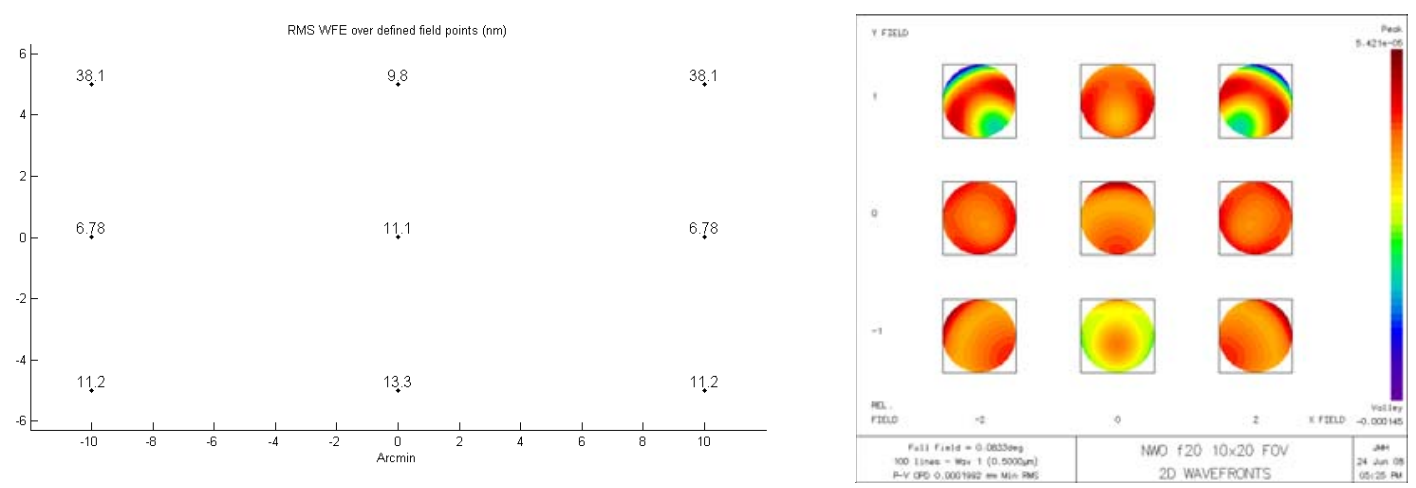

Figure 5. Optical performance in RMS WFE (nm) at the TMA Focal Surface for the initial design.

It is important to note that the closed-form analytical solution for the TMA design as defined by D. Korsch does NOT correct image quality at the internal focus. This Cassegrain focus (CF) is highly aberrated such that the tertiary mirror (TM) corrects the light at the image surface of the TMA, as shown in Figure 6, where the spherical aberration is approximately $58 \mathrm{~nm}$ RMS WFE on the axis of the CF. Further inspection in the field of the CF will show spherical, coma, and astigmatism (and field curvature).

To correct this spherical aberration, i.e. step 2, simply vary the radius and conic constant of the primary mirror, and optimize the system with a downhill method at the axial field point. The CF will rapidly converge in image quality, as shown in Figure 7, where the new residual on axis is $\sim 1 \mathrm{~nm}$ RMS WFE. At this point, one would expect the image quality of the TMA to degrade, and it does, as clearly apparent in Figure 8, where the maximum WFE across the FOV is now almost $250 \mathrm{~nm}$ RMS WFE. Note that the form of the WFE is dominated by spherical aberration, and is nearly uniform across the field. This makes sense since it is the PM that is modified, which is the aperture stop of the system. Since there is no beam wander across the PM as a function of field, any aberration introduced by the PM will be uniform as a function of field.

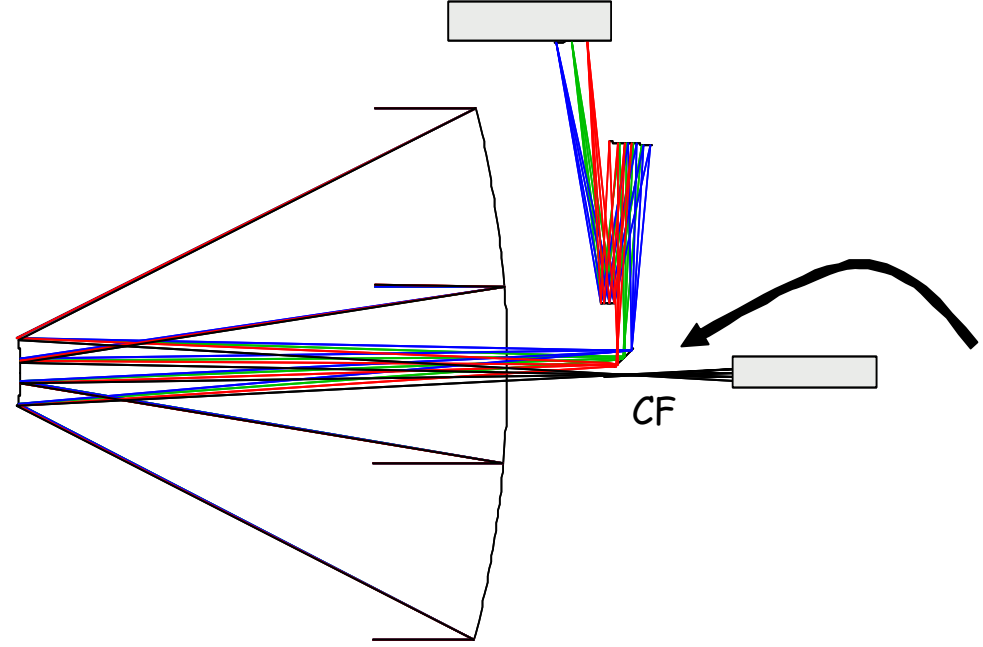

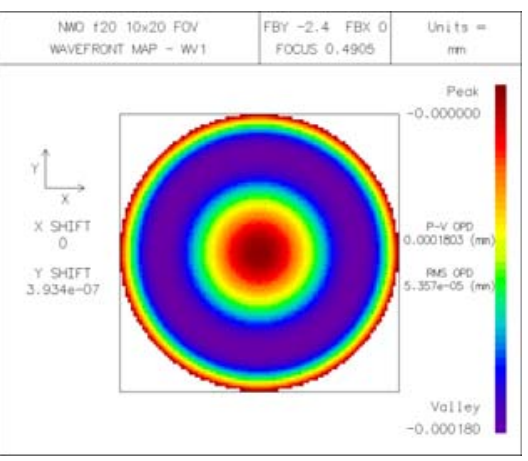

$58 \mathrm{~nm}$ RMS WFE

Figure 6. Optical performance at the Cassegrain Focus (CF) for the initial design. 


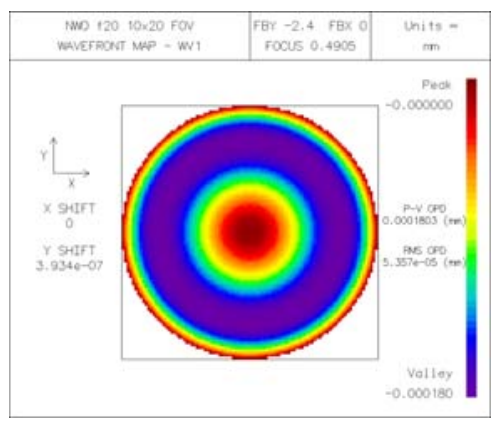

$58 \mathrm{~nm}$ RMS WFE

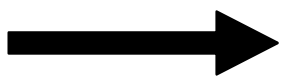

Figure 7. Correction of RMS WFE at CF using PM figure

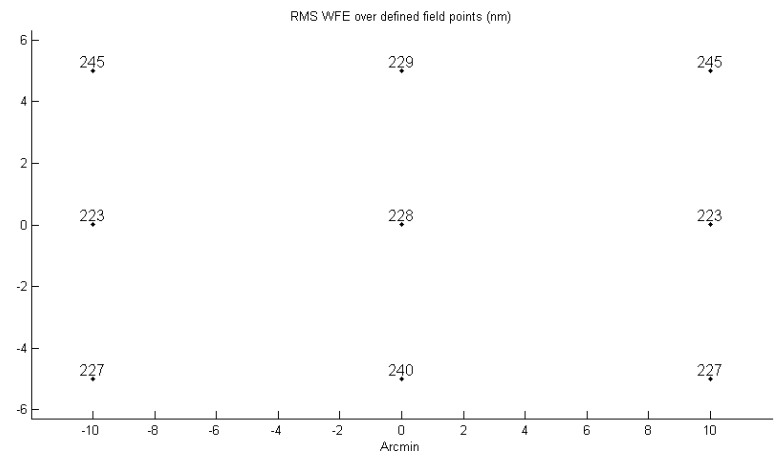

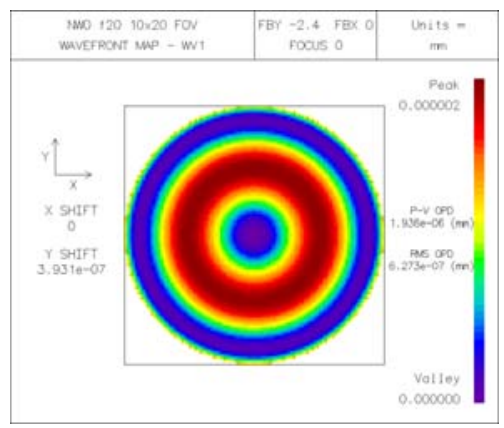

$\sim 1 \mathrm{~nm}$ RMS WFE

Figure 8. Resulting optical performance at TMA Focal Surface after PM correction of CF.

In step 3 and 4, the goal is to keep the CF performance as is, while returning the TMA image quality to its previous nominal design residual (or even perhaps improve it, if possible). Since the PM and SM are now fixed, the obvious method is to first see how far varying the TM will improve the matter. Figure 9 shows improvement of the system down to about $90 \mathrm{~nm}$ RMS WFE, where the form of the residual WFE is still fairly constant across the FOV (i.e. spherical aberration dominated). The final step is to allow an aspheric deformation on a flat fold mirror at the exit pupil of the telescope, i.e the FM in Figure 4. This mirror is conjugate to the PM, so its location is ideal to act as a "corrector" to the "perturbed" PM prescription. After varying just the fourth order aspheric coefficient on the mirror (while keeping the curvature flat), downhill optimization greatly improves the TMA image surface quality to that shown in Figure 9. The final result is nearly identical to that shown in the "nominal" TMA starting design shown in Figure 5.
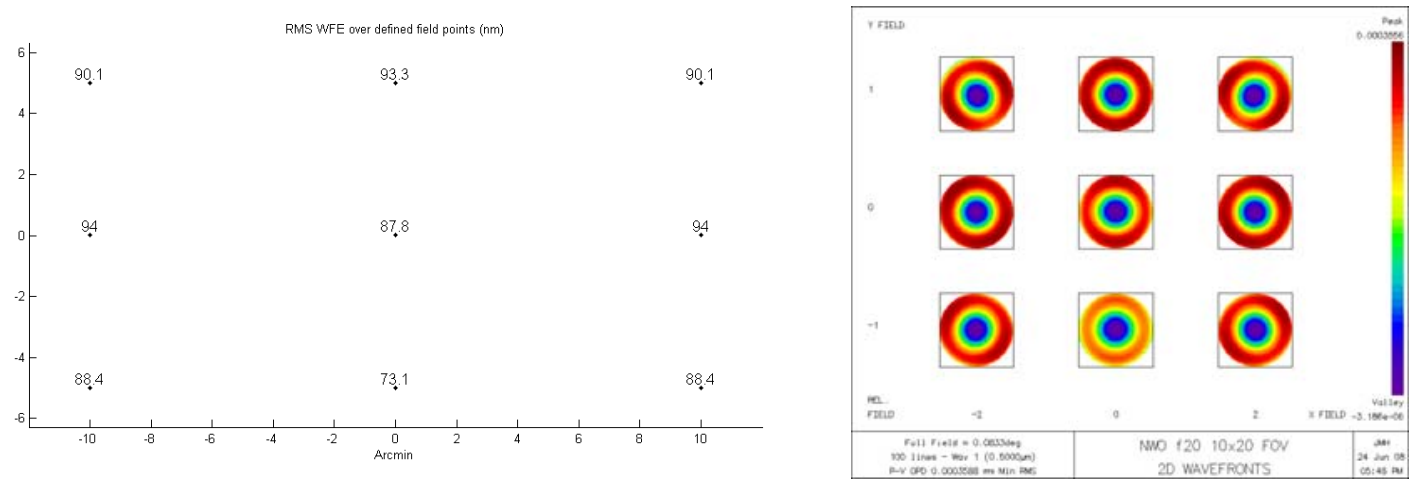
Figure 9. Improvement in optical performance at TMA Focal Surface after TM optimization.
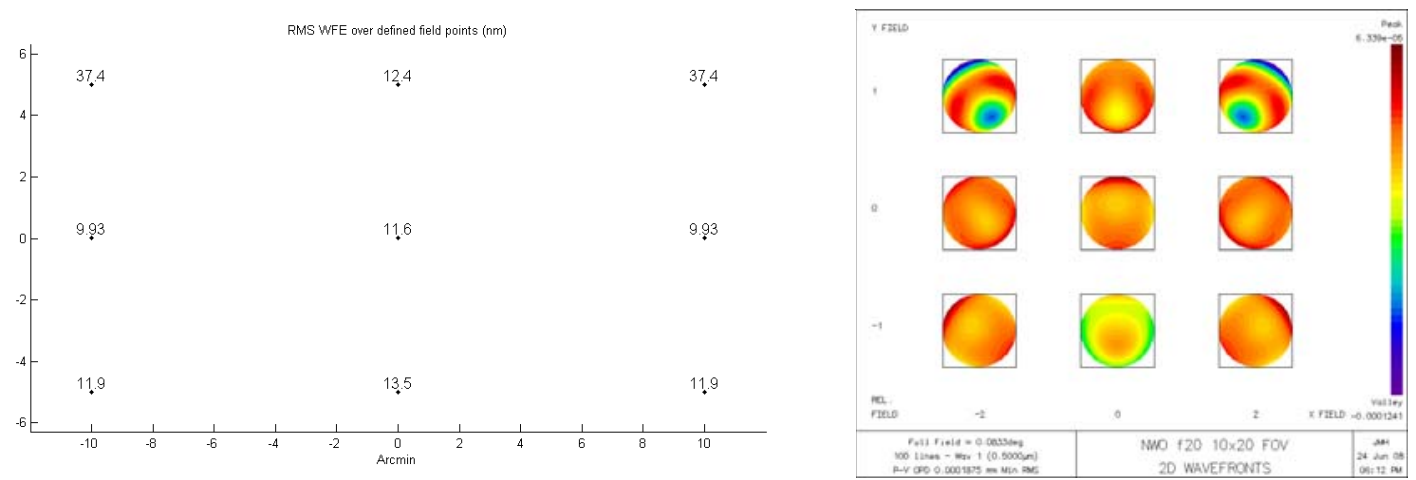

Figure 10. Final optical performance at TMA Focal Surface after FM optimization. The result here is nearly identical to the starting design shown in Figure 5.

Note that it is possible to also get very good correction by ignoring step 3, and using the FM only to correct the system. Such a modification and correction to the design can be compared with the PM fabrication error on the Hubble Space Telescope, and the resulting correction to full performance with the COSTAR optics [8].

While the TMA image quality is nearly unchanged from the initial design (i.e. corrected for third-order spherical, coma, and astigmatism), a close investigation of the image quality around the $\mathrm{CF}$ will show that it is dominated by third-order coma, as show in Figure 11. The field shown extends to a full arc-minute, while the magnitude of the RMS WFE increases to just under $4 \mathrm{~nm}$. This form of error is typical of a two-mirror Cassegrain or Gregorian telescope design where the primary mirror is essentially a parabola, and the secondary relays the perfect focus on axis to a final image surface, giving spherical correction through all orders. Note that the PM conic for this TMA given here is not a parabola (but it is close).
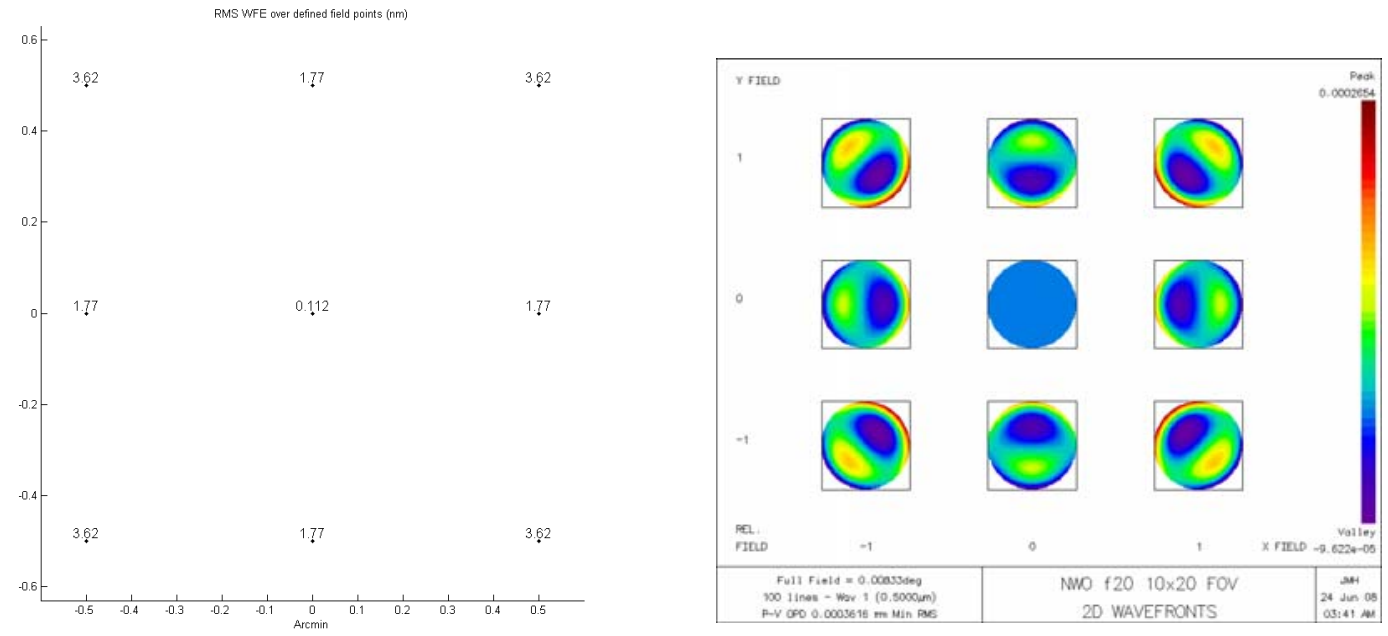

Figure 11. Final optical performance at Cassegrain Focus (CF) after using PM to correct, over 1 arc-minute field of view.

One would naturally believe that improved field performance of the CF can be achieved by allowing the SM to vary in radius and conic, resulting in a "Ritchey-Chretien" correction of both spherical and coma at the CF. This is true, but the resulting image quality at the TMA focal surface can prove disastrous. In Figure 12, the PM and SM were varied to allow correction at the CF over a 1 arcmin FOV, which resulted in very good performance at the CF. 
When the TMA was corrected with the TM and FM, however, one can see that the center of the field is corrected well, but the edges are dominated by both coma and astigmatism. Since the FM is not conjugate to the SM, it cannot fully correct a "perturbation" across the field, and hence is not suggested as a design approach for this form of telescope.
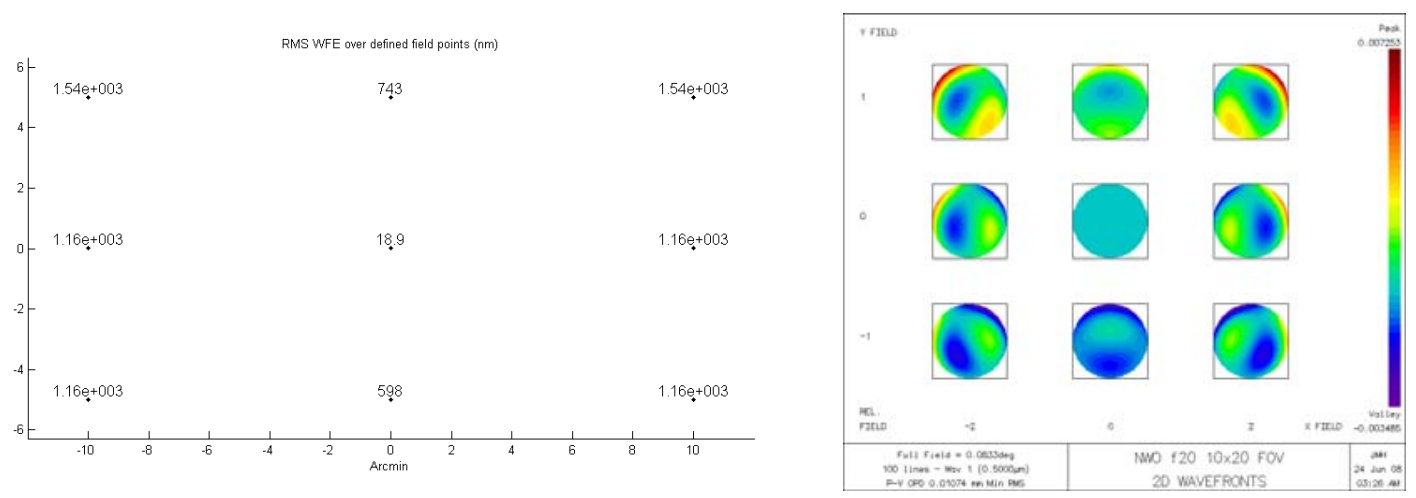

Figure 12. Final optical performance at TMA Focal Surface after using PM and SM to correct CF, then TM and FM optimization to correct TMA Focal Surface.

\section{CONCLUDING REMARKS}

The process outlined above has proved successful for several design concepts for NWO, included the baseline presented here. Naturally, a rigorous approach towards constraining the dual-channel telescope would be to further constrain the Korsch equations for a TMA to further constrain the spherical aberration at the CF. Casual inspection of those equations suggests that the analytical solution (if it exists) will be quite a challenge to solve for, and perhaps appropriate for an enterprising graduate student in optical design to attempt. A numerical approach is certainly more tenable, as demonstrated indirectly in this work, and may be the subject of a more thorough future paper on the topic.

\section{REFERENCES}

[1] Bulletin of the American Astronomical Society, Vol. 37, p.1357.

[2] Please search for "CorSPEC" on www.elsevier.com/locate/newastrev

[3] A summary of MAG30Cam can be downloaded from: http://sco.stsci.edu/tpf downloads/mag30cam july21.pdf

[4] "Finding Earth-size planets in the habitable zone: the Kepler Mission," Borucki et. al., Proc IAU, Volume 249,

p. 17-24.

[5] D. Korsch, "Closed Form Solution for Three-Mirror Telescopes, Corrected for Spherical Aberration, Coma, Astigmatism, and Field Curvature," Appl. Opt. 11, 2986-2987 (1972).

[6] Joseph M. Howard, "SLIDERS: the next generation of automated optical design tools has arrived", Proc. SPIE 5174, 19 (2003). SLIDERS can either be downloaded from the Lambda Research Corp. website after registering with them, or by contacting the author via email at $<$ Joe.Howard@nasa.gov $>$.

[7] OSLO is a trademark from Lambda Research Corporation, Littleton, MA, USA. A free copy of OSLO EDU can be downloaded from the internet at: http://www.lambdares.com/education/oslo edu/

[8] M. Bottema, "Relective correctors for the Hubble Space Telescope axial instruments," Appl. Opt. 32, 1768(1993). 